\title{
Holographic coupler-optical fiber system: mathematical model for the coupling optimization
}

\author{
Maria L. Calvo and L. De Pedraza
}

\begin{abstract}
A mathematical model for describing a holographic coupler-optical fiber system is considered. The object wave to be recorded in the hologram is assumed to be a polarized wave which is scattered by the optical fiber. In the reconstruction the optimal condition for the diffracted wavefront to be coupled to a second fiber is obtained and numerically analyzed.
\end{abstract}

\section{Introduction}

Optical fibers are widely used for signal transmission at small, middle, and large distances due to their large capability for confinement and propagation of information with low loss. In the design of particular optical and optoelectronic systems where optical fibers are required, fiber to fiber connections are sometimes required. ${ }^{1}$ Connectors based on the use of holographic elements have been developed for the coupling of a pair of single-mode fibers. ${ }^{2}$ The method is applicable to a bundle of fibers as well.

The efficiency of the transfer of energy from the first fiber to the second depends on the recording conditions and optical parameters of the fiber. This second aspect gives rise to an intrinsic coupling efficiency. To connect two fibers with different optical parameters a compound holocoupler formed by two aligned hololenses is used. A first holographic recording is made with the output signals coming from the end of both fibers. In the recording of the second hologram the conjugated reference wave of the first is usually chosen to assure coupling when the experimental setup is aligned.

Let the total efficiency be defined as the product of the transmission efficiency of the hologram $\epsilon_{T}$ times the intrinsic fiber efficiency $\epsilon_{i}$. Then values of $\sim 0.25$ were found for the holocoupler-optical fiber system $\left(\epsilon_{T}\right.$ $\times \epsilon_{i}=0.65 \times 0.39=0.25$ ), which can be compared with a value of 0.24 for a $10 \times$ microscope objective $\left(\epsilon_{T} \times \epsilon_{i}=\right.$ $0.36 \times 0.67=0.24$ ).

The authors are with Complutense University, Faculty of Physical Sciences, Optics Department, 28040 Madrid, Spain.

Received 12 February 1988.

$0003-6935 / 89 / 112031-08 \$ 02.00 / 0$.

(C) 1989 Optical Society of America.
Several other authors have developed various types of transmission holocoupler for various purposes, such as in optical integrated circuits, ${ }^{3,4}$ branching holocouplers, ${ }^{5}$ holocouplers for single-mode fibers, ${ }^{6}$ multiple holocouplers, ${ }^{2}$ as well as reflection holocouplers. ${ }^{7}$

Generally speaking, recording is optimized by using a volume hologram allowing the storage of a large amount of information. The maximum number of exposures supported by a volume hologram can be estimated through Kogelnik's theory. ${ }^{8}$ For photopolymers as holographic materials and a $100 \%$ efficiency, ten exposures are required. ${ }^{9}$ In multiple exposures nonlinear effects must be avoided. ${ }^{10,11}$

Theoretical formulations of mechanisms for coupling the energy from one slab guide into another were developed by Leite et al., ${ }^{12}$ who calculated the impulse response of the optical system. A source function was defined through the Fresnel integral. Within the limits of the paraxial approximation a complete formulation of the transfer characteristics of the double hologram coupler was obtained. From this formulation the intrinsic coupling efficiency was defined for a particular mode of the propagated field.

The aim of the present work was to search for a simple mathematical model to describe the mechanism for the coupling of energy in a holographic coupler-optical fiber system. For that purpose we have made use of a previously established formulation for the field scattered by an optical fiber. ${ }^{13}$ We have assumed that the incoming electric field striking the fiber is polarized parallel to its axis (TM wave). This scattered field is expressed in terms of an integral equation which can be simplified through an eikonal solution (in the sense of Glauber's approximation ${ }^{14}$ ) and acts as the object wave in the hologram recording process. The conditions for the validity of this approximation have been checked, thereby providing support to earlier analogous treatments proposed by 


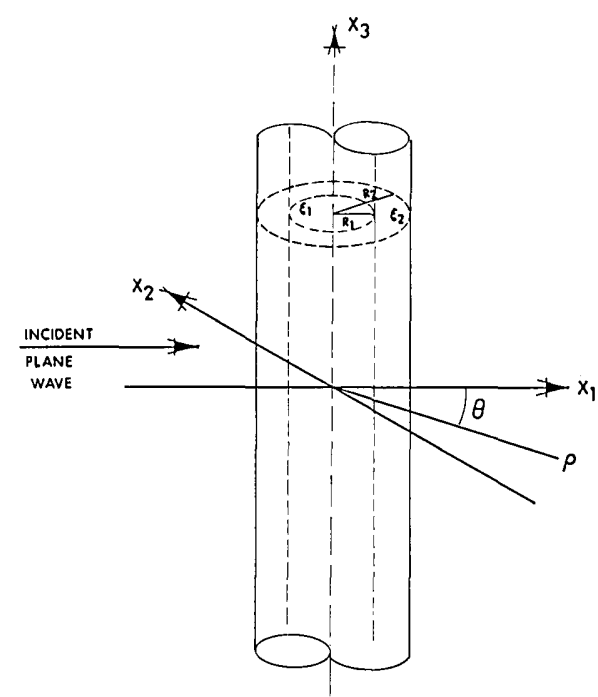

Fig. 1. Lateral illumination of the optical fiber and physical parameters.

other authors..$^{15}$ The transmittance of the recorded hologram is defined, and a final condition is obtained for the reconstruction process. This is expressed as an inequality representing the compensation in the phase of the field diffracted by the hologram at the entrance face of the second fiber. Such an interpretation is equivalent to establishing a boundary condition at this plane.

\section{Theoretical Background: Eikonal Approximation for the Light Scattered by an Optical Waveguide}

We consider the scattering of a classical monochromatic electromagnetic (em) wave by an inhomogeneous dielectric optical waveguide of infinite length. The latter is parallel to the $X_{3}$ axis and has an arbitrary cross section $\Omega$, and it is characterized by a variable dielectric permittivity $\epsilon(\rho)$ (see Fig. 1). Here $\rho=$ $\left(X_{1}, X_{2}\right)$ represents the position of a point in the $X_{1} X_{2}$ plane orthogonal to $X_{3}$. The incoming em wave orthogonal to the $X_{3}$ axis is represented by the electric field $\left[0,0, E_{0} \exp (i \mathbf{K} \rho)\right]$ with wave vector $\mathbf{K}=\left(K_{1}, K_{2}\right), K$ $=\left[\mathbf{K}^{2}\right]^{1 / 2}>0$.

The case of an electric field polarization parallel to the fiber axis is useful for the determination of the fiber parameters. ${ }^{15}$ The general differential wave equation reduces to the following for the total electric field $[0,0, E(\rho)]$, which is parallel to $X_{3}$ (TM wave):

$$
\left(\Delta_{p}+K^{2}\right) E= \begin{cases}0, & \rho \text { outside } \Omega, \\ -K^{2}(\epsilon-1) E, & \rho \text { inside } \Omega,\end{cases}
$$

with $\Delta_{\rho}=\partial^{2} / \partial X_{1}^{2}+\partial^{2} / \partial X_{2}^{2}$. Knowledge of the incoming wave and Eq. (1) lead using standard techniques to the scattering integral equation

$$
\begin{aligned}
E(\rho)= & E_{0} \exp (i \mathrm{~K} \rho)-(1 / 4) \int_{\Omega} d^{2} \rho^{\prime} H_{0}^{(1)} \\
& \times\left(K, \rho-\rho^{\prime}\right) K^{2}\left[\epsilon\left(\rho^{\prime}\right)-1\right] E\left(\rho^{\prime}\right)
\end{aligned}
$$

$\left(H_{(1)}^{0}\right.$ is Hankel's function of first kind and zeroth order), where both $\rho=\left(X_{1}, X_{2}\right)$ and $\rho^{\prime}=\left(X_{1}^{\prime}, X_{2}^{\prime}\right)$ are the two references points in the $X_{1} X_{2}$ plane perpendicular to the $X_{3}$ axis, $\Omega$ is the cross section of the optical fiber (see Fig. 1), and $\epsilon(\rho)=n^{2}$ is the dielectric permittivity of the fiber.

An approximate analytical solution for the exact scattered field formulated in Eq. (2) can be obtained through the so-called eikonal approach (in the sense of Glauber's approximation ${ }^{15}$ ), which describes the scalar field diffracted at the output plane of the optical waveguide. Assuming that the validity conditions of this approximation hold for both small and large diameters of the core, the numerical analysis for $E(\rho)$ simplifies dramatically.

The first condition is that the product of the modulus of the incoming wave vector $|\mathbf{K}|=K$ and the total radius of the fiber $R_{2}$ must be larger than unity: $K R_{2}$ $\gg 1$; that is, the wavelength of the incoming radiation is much smaller than $R_{1}$.

We define $E_{\text {eik }}^{\prime}(\rho)$ by the integral equation [compare with Eq. (2)]

$$
\begin{aligned}
E_{\text {eik }}^{\prime}(\rho)= & E_{0} \exp (i \mathrm{~K} \rho)-(1 / 4) \\
& \times \int_{\Omega} d^{2} \rho^{\prime} H_{0, \mathrm{eik}}^{(1)}\left(K, \rho-\rho^{\prime}\right) K^{2}\left[\epsilon\left(\rho^{\prime}\right)-1\right] E_{\text {eik }}^{\prime}\left(\rho^{\prime}\right),
\end{aligned}
$$

where

$$
\begin{aligned}
H_{0, \mathrm{eik}}^{(1)}\left(\mathbf{K}, \rho-\rho^{\prime}\right)= & (2 / K) \exp \left[i k\left(X_{1}-X_{1}^{\prime}\right)\right] \\
& \times \theta\left(X_{1}-X_{1}^{\prime}\right) \delta\left(X_{2}-X_{2}^{\prime}\right),
\end{aligned}
$$

where $\theta$ and $\delta$ are the step and Dirac delta functions, respectively.

To obtain the scattering amplitude $T\left(\mathbf{K}^{\prime}, \mathbf{K}\right)$, we shall consider the asymptotic behavior of $H_{0}^{(1)}$ in Eq. (2). Then for fixed $\rho /|\rho|$ and $|\rho| \rightarrow \infty\left(\left|\rho-\rho^{\prime}\right| \sim|\rho|-\mathbf{K}^{\prime}\right.$. $\left.\boldsymbol{\rho}^{\prime}, \mathbf{K}^{\prime}=(K \cdot \rho) /|\rho|\right)$, Eq. (2) yields

$$
E(\rho) \sim E_{0}\left(\exp (i \mathbf{K} \boldsymbol{\rho})+\left[\exp (i K|\rho|) /|\rho|^{1 / 2}\right] \cdot T\left(\mathbf{K}^{\prime}, \mathbf{K}\right)\right\} .
$$

The scattering amplitude in the eikonal approxima$\operatorname{tion}^{16}$ is

$$
\begin{aligned}
T_{\text {eik }}\left(\mathbf{K}^{\prime}, \mathbf{K}\right)= & (K / 2 \pi)^{1 / 2} \exp (-i \pi / 4) \int_{-\infty}^{+\infty} d X_{2}^{\prime} \exp \left[-i K \operatorname{sen}(\theta) X_{2}^{\prime}\right] \\
& \times\left\{\exp \left[i \varphi_{\text {eik }}\left(X_{1}=+\infty, X_{2}^{\prime}\right)\right]-1\right\}
\end{aligned}
$$

where

$$
\varphi_{\text {eik }}\left(X_{1}, X_{2}\right)=(K / 2) \int_{-\infty}^{X_{1}} d X_{1}^{\prime}\left[\epsilon\left(X_{1}^{\prime}, X_{2}\right)-1\right]
$$

is the phase of the scattered wave expressed in the eikonal approximation and $\theta$ is the scattering angle (see Fig. 1).

One expects $T_{\text {eik }}$ to be a good approximation to the exact scattering amplitude $T$ when $R_{1} K|1-\cos \theta|$ is small compared to 1 .

If we consider that the refractive index distribution of the optical fiber has a parabolic profile; the eikonal phase has the following explicit values for regions inside and outside the core:

$$
\begin{aligned}
& \varphi_{\text {eik }}=0 \text {; } \\
& \left|X_{2}\right|>R_{2} \text {, } \\
& \varphi_{\text {eik }}=K\left(\epsilon_{2}-1\right)\left(R_{2}^{2}-X_{2}^{2}\right)^{1 / 2} \text {; } \\
& R_{2}>\left|X_{2}\right|>R_{1} \text {, }
\end{aligned}
$$




$$
\begin{array}{rlrl}
\varphi_{\text {eik }}= & K\left\{\left(\epsilon_{2}-1\right)\left[\left(R_{2}^{2}-X_{2}^{2}\right)^{1 / 2}-\left(R_{1}^{2}-X_{1}^{2}\right)^{1 / 2}\right]\right. \\
& +\left(R_{1}^{2}-X_{2}^{2}\right)^{1 / 2}\left[\left(\epsilon_{2}-1\right)-\left(\Delta \epsilon_{1} / 3 R_{1}^{2}\right)\right. & \\
& \left.\left.\times\left(R_{1}^{2}+2 X_{2}^{2}\right)\right]\right\} ; \quad R_{1}>\left|X_{2}\right|>0,
\end{array}
$$

where $\epsilon_{1}$ denotes the dielectric permittivity for the core, while $\epsilon_{2}$ is that for the cladding of the fiber. $R_{1}$ is the radius of the core, and $R_{2}$ is the total radius of the fiber. The coupling constant $\Delta$ is defined as

$$
\Delta=\left(\epsilon_{1}-\epsilon_{2}\right) / 2 \epsilon_{1} .
$$

Thus there are two intervals defined inside and outside the core (inside the cladding) for which the eikonal phase $\varphi_{\text {eik }}$ is well defined. Then, according to Eq. (6), the eikonal scattering amplitude for the parabolic profile is

$$
\begin{aligned}
T_{\text {eik }}\left(\mathbf{K}^{\prime}, \mathbf{K}\right)= & (2 K / \pi)^{1 / 2} \exp (-i \pi / 4)\left(\int_{0}^{+R_{1}} d X_{2}^{\prime} \cos \left[K \operatorname{sen}(\theta) X_{2}^{\prime}\right]\right. \\
& \times\left\{\exp \left[i \varphi_{\text {eik }}\left(X_{2}^{\prime}\right)\right]-1\right\} \\
& +\int_{+R_{1}}^{+R_{2}} d X_{2}^{\prime} \cos \left[K \operatorname{sen}(\theta) X_{2}^{\prime}\right] \\
& \left.\times\left\{\exp \left[i \varphi_{\text {eik }}\left(X_{2}^{\prime}\right)\right]-1\right\}\right)
\end{aligned}
$$

which can be directly evaluated by using Eqs. (8)-(10).

\section{A. Validity of the Approximation}

To estimate the validity of the eikonal approximation in the scattering amplitude for the parabolic profile we make use of the following dimensionless quantity ${ }^{13}$ :

$$
\begin{aligned}
& \nu=(1 / 4)\left(\epsilon_{2}-1\right) \text { for } R_{2}>\left|X_{2}\right|>R_{1},|\rho|<R_{2} \text { or } \\
& \text { for } R_{1}>\left|X_{2}\right| \geqslant 0, R_{2}>|\rho|>R_{1}, X_{1}<0 ;
\end{aligned}
$$

also

$$
\begin{aligned}
\nu=N_{2}= & (1 / 4)\left(\epsilon_{2}-1\right)+\left[\epsilon_{1} \Delta / K R_{1}\left(\epsilon_{2}-1\right)\right] \\
& \times\left[\lambda\left(R_{2}, X_{2}\right)+\lambda\left(R_{1}, X_{2}\right)\right] \\
& +\left[\epsilon_{1}^{2} \Delta^{2}\left(X_{2} / R_{1}\right)^{2} /\left(\epsilon_{2}-1\right)\right]\left[\lambda\left(R_{2}, X_{2}\right)+\lambda\left(R_{1}, X_{2}\right)\right]^{2}
\end{aligned}
$$

for the interval $R_{1}>\left|X_{2}\right| \geqslant 0, R_{2}>|\rho|>R_{1}, X_{1}>0$, and

$$
\begin{aligned}
\nu=N_{1}= & (1 / 4)\left[\epsilon_{1}-1-\Delta\left(|\rho| / R_{1}\right)^{2}\right] \\
& +\left\{\epsilon_{1} \Delta\left(\left|X_{1}\right| / R_{1}\right) / K R_{1}\left[\epsilon_{1}-1-\Delta\left(|\rho| / R_{1}\right)^{2}\right]\right\} \\
& +\left\{\epsilon_{1} \Delta\left[\lambda\left(R, X_{2}\right)+X_{1} / R_{1}\right] / K R_{1}\left[\epsilon_{1}-1-\Delta\left(|\rho| / R_{1}\right)^{2}\right]\right\} \\
& +\left\{\epsilon _ { 1 } ^ { 2 } \Delta ^ { 2 } ( X _ { 2 } / R _ { 1 } ) ^ { 2 } \left[\lambda\left(R_{2}, X_{2}\right)\right.\right. \\
& \left.\left.+X_{1} / R_{1}\right]^{2} /\left[\epsilon_{1}-1-\Delta\left(|\rho| / R_{1}\right)^{2}\right]\right\}
\end{aligned}
$$

for the interval $R_{1}>\left|X_{2}\right|>0,|\rho|<R_{1}$, where in Eqs. (13)-(14) the parameter $\lambda$ is $\lambda(\alpha, \beta)=\left[\left(\alpha / R_{1}\right)^{2}-(\beta /\right.$ $\left.\left.R_{1}\right)^{2}\right]^{1 / 2}$. The eikonal approximation holds if $\nu<1$ for all the above regions.

To estimate the validity of the approximation we made a numerical computation of Eqs. (12)-(14), and then we calculated the bounds for the parameter $\nu$. The physical parameters introduced are a fixed value for the total radius of the fiber $\left(R_{2}=100 \mu \mathrm{m}\right)$ and a variable parameter for the internal radius of the core

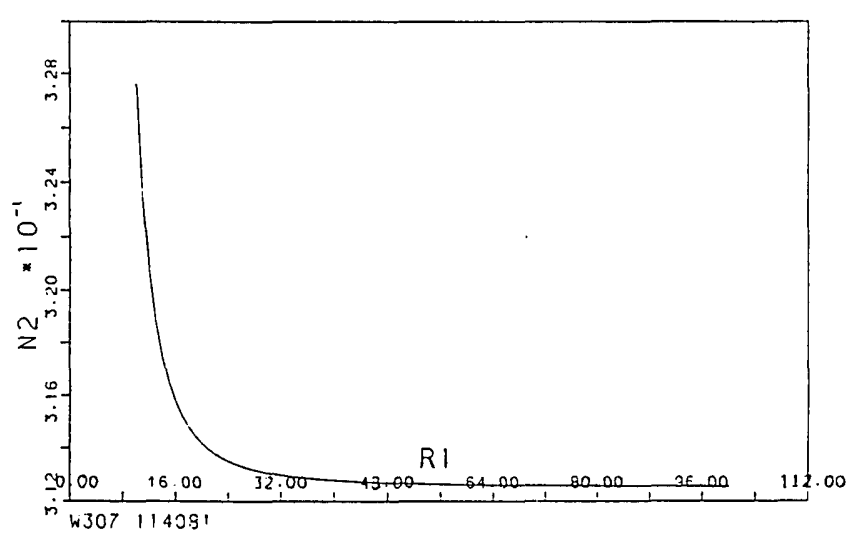

(a)

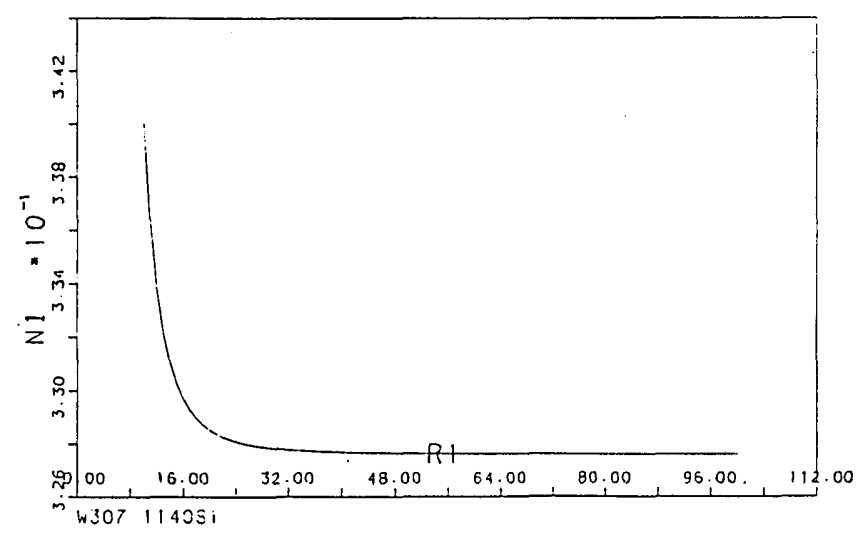

(b)

Fig. 2. Validity of the eikonal approximation for a TM wave scattered by an optical fiber: (a) interval defined as $\left.R_{1}\right\rangle\left|X_{2}\right| \geqslant 0, R_{2}>$ $|\rho|>R_{1}$ with $X_{1}>0$. (b) Interval defined as $|\rho|<R_{1}, R_{1}>\left|X_{2}\right| \geqslant 0$.

$\left(10 \mu \mathrm{m}<R_{1}<100 \mu \mathrm{m}\right)$. Also $\epsilon_{2}=2.25$ and $\epsilon_{1}=2.3104$, so that the constant $\Delta$ is of the order of 0.01 .

The numerical results are shown in Fig. 2. Figure 2(a) represents the values for $\nu=N_{2}$ [see Eq. (13)], with $0.31<\nu<0.33$, and Fig. 2(b) represents the values for $\nu$ $=N_{1}$ [see Eq. (14)] with $0.33<\nu<0.34$. We can observe in both figures the large region of validity for the eikonal approximation for fixed values of the coordinates $X_{1}$ and $X_{2}$.

The vertical axis shows the values obtained for the coefficient describing the validity of the approximation, while the horizontal axis shows the possible values for the internal radius of the fiber. It is also observed that the larger the internal radius the better the validity of the approximation. Thus the scattering considered in the eikonal approximation is valid for both thin and thick fibers.

\section{Mathematical Model: Coupling Condition in a Holocoupler-Optical Fiber System}

We made use, for the purpose of developing our mathematical model, of the experimental setup shown in Fig. 3 for recording a holocoupler. ${ }^{2,17}$ In general, the 


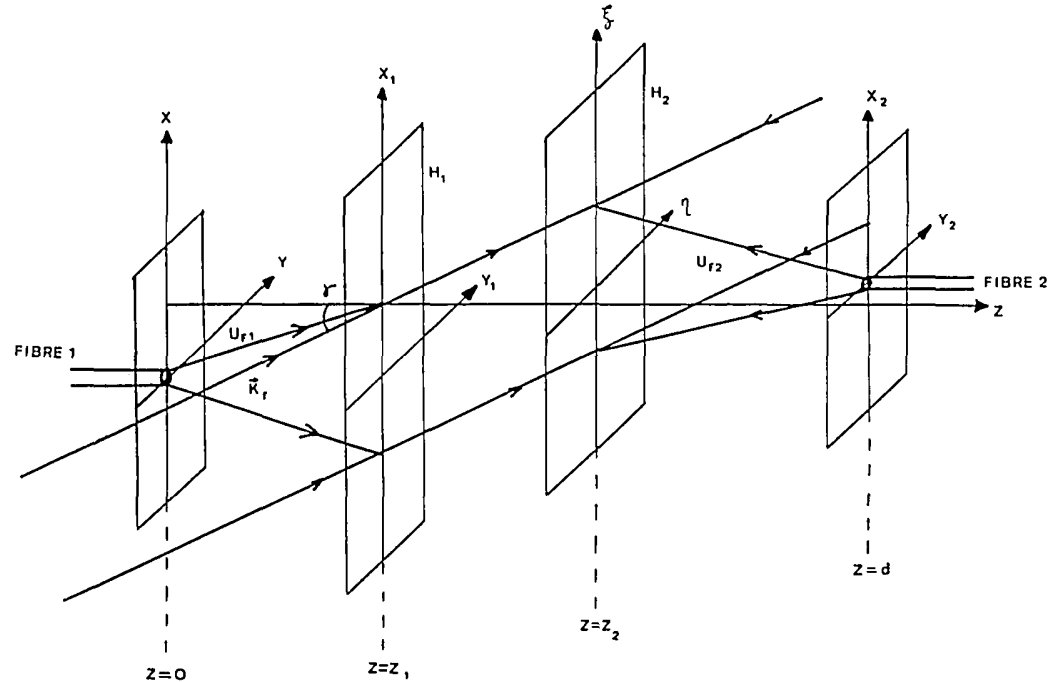

holographic coupler is an interferogram formed as follows: (1) the first hologram $H_{1}$ placed in the plane $Z=$ $Z_{1}$ is recorded with the output beam of the optical fiber 1 (defined at plane $Z=0$ ) and the incoming reference beam considered as a plane monochromatic wave; and (2) the second hologram $H_{2}$ located in plane $Z=Z_{2}$ is recorded by the output beam of fiber 2 (defined at plane $Z=d$ ) and the conjugate of the first reference wave.

In the best condition for coupling, if we reconstruct with the output from fiber 1 , the light diffracted by the holocoupler is transmitted and focused into fiber 2.

Our major interest is the search of an analytical expression for the field generated at the aperture of fiber 2 after the reconstruction process mentioned above. For that we shall make use of the previously established mathematical expression for the scattered signal in the eikonal approximation.

According to the scheme for the recording process displayed in Fig. 3, we shall define the following fields:

$$
\begin{aligned}
U_{F 1} & =\text { O.O. (1), } \\
U_{R 1} & =\text { O.R. (1), }
\end{aligned}
$$

where $U_{F 1}$ is the object wave of the first fiber and $U_{R 1}$ the reference wave, and

$$
\begin{aligned}
U_{F 2} & =\text { O.O. (2), } \\
U_{R 2} & =\text { O.R. (2), }
\end{aligned}
$$

are the object and reference waves of the second fiber, respectively.

$U_{F 1}$ and $U_{F 2}$ are scattered signals coming from the apertures of fibers 1 and 2, respectively, while $U_{R 1}$ and $U_{R 2}$ are plane monochromatic waves. Also, we shall consider that the holocoupler is recorded in linear conditions.

The transmitted field by the first hologram denoted by $U_{c}$ is

with

with
Fig. 3. Holographic coupler: schematic representation of the experimental setup for the recording process.

$$
U_{\mathrm{c}}=U_{F 1} T_{1}
$$

$$
T_{1} \sim\left|U_{F 1}+U_{R 1}\right|^{2}
$$

being the amplitude of the transmission field of the first hologram.

Accordingly, the transmitted field by the second hologram is denoted by $U_{c} T_{2}$ :

$$
\begin{aligned}
U_{c} T_{2} & =U_{F 2}^{*}, \\
U_{R 2} & =U_{R 1}^{*},
\end{aligned}
$$

$$
T_{2} \sim\left|U_{F 2}+U_{R 2}\right|^{2}
$$

being the amplitude of the transmission field of the second hologram.

If we obtain the real image for the first hologram and consider that optical filtering is applied to the intensity terms, $U_{F 1}\left(U_{F 1}^{2}+U_{R 1}^{2}\right)$ and $U_{c}\left(U_{F 2}^{2}+U_{R 2}^{2}\right)$, then

$$
U_{c} \sim U_{F 1} U_{R 1} U_{F 1}^{*} \text {, }
$$

so that the final condition is

$$
\left(U_{R 2}^{*}\right)^{2}\left|U_{F 1}\right|^{2} U_{F 2}=\left(1-R^{2}\left|U_{F 1}\right|^{2}\right) U_{F 2}^{*},
$$

where $R^{2}=\left|U_{R 1}\right|^{2}=\left|U_{R 2}\right|^{2}$ is the square of the amplitude of the reference wave.

According to Eqs. (5)-(7), we shall define the scalar field diffracted at the output plane of fibers 1 and $2^{16}$ as

$$
\begin{aligned}
U_{F j} & \sim \exp \left[(-1)^{j+1} i \varphi_{\text {eik }}\left(X_{2 j}\right)\right]-1 ; \quad j=1,2, \\
\left|U_{F j}\right|^{2} & \sim 2\left\{1-\cos \left[\varphi_{\text {eik }}\left(x_{2 j}\right)\right]\right\},
\end{aligned}
$$

where $X_{21}=X_{2}^{\prime}$ is the vertical coordinate defined at the aperture of fiber 1 and $X_{22}=X_{2}$ is the vertical coordinate at the aperture of fiber 2 . Here $U_{F 1}$ is the wave propagating to the right from fiber 1 , while $U_{F 2}$ is the wave propagating to the left from fiber 2 (see Fig. 3).

Equation (23) together with Eqs. (24)-(25) forms a system of implicit functions in the vertical coordinates 
$X_{21}$ and $X_{22}$ at the output planes of fibers 1 and 2 . We have established the necessary and sufficient condition for the system to have a nontrivial solution different from zero. For that the Jacobian of the system $J$ must be different from zero (see Appendix A for details of this calculation). After performing some manipulations we arrive at the following inequality:

$$
\begin{aligned}
\cos \left[\varphi_{\text {eik }}\left(X_{2}\right)\right]+\cos \left[-\varphi_{\text {eik }}\left(X_{2}\right)+2 \mathbf{K}_{R} \cdot \mathbf{r}\right] \\
\quad \neq(1 / 2)\left\{\cos 2\left[-\varphi_{\text {eik }}\left(X_{2}\right)+\mathbf{K}_{R} \cdot \mathbf{r}\right]+\cos \left(2 \mathbf{K}_{R} \cdot \mathbf{r}\right)\right\}+1 .
\end{aligned}
$$

For simplicity we shall denote

$$
\begin{aligned}
& P=\cos \left[\varphi_{\mathrm{eik}}\left(X_{2}\right)\right]+\cos \left[-\varphi_{\mathrm{eik}}\left(X_{2}\right)+2 \mathbf{K}_{R} \cdot \mathbf{r}\right], \\
& S=(1 / 2)\left\{\cos 2\left[-\varphi_{\mathrm{eik}}\left(X_{2}\right)+\mathbf{K}_{R} \cdot \mathbf{r}\right]+\cos \left[2 \mathbf{K}_{R} \cdot \mathbf{r}\right]\right\}+1 .
\end{aligned}
$$

According to the mathematical method developed in Appendix A two possible cases are considered:

(a) $P$ and $S$ are functionally independent. This is obtained from the condition where the Jacobian of the system is different from zero $(J \neq 0)$. Then the coupling condition holds.

(b) $P$ and $S$ are functionally dependent. This results from the condition where the Jacobian of the system is equal to zero $(J=0)$. Then there is no coupling in the system.

We notice that the system represented in Eq. (A3) physically means the point at which the two curves $F\left(X_{21}, X_{22}\right)$ and $G\left(X_{21}, X_{22}\right)$ [see Eqs. (A4)-(A5)] intersect. The condition that the Jacobian of the system is different from zero implies that the tangents to both curves at the intersection point are not parallel. In our case we obtain the value of the phase modulation of the field entering fiber 2 required for energy transfer.

The inequality expresses, within the limits of the eikonal approximation, the coupling condition in terms of the vertical coordinate $X_{2}$ of the aperture pupil of the second fiber and the phase of the reference wave $\mathbf{K}_{R} \cdot \mathbf{r}$. We can explore the tolerance in terms of these parameters.

\section{A. Coupling Efficiency}

Let us assume that the mode in fiber 2 is represented by $U_{F 2}$ as given in Eq. (24). The coupling efficiency $\epsilon$ is then defined as

$$
\epsilon=\left|\int_{R_{1}}^{R_{2}} d X_{2} U_{F 2}\right|^{2},
$$

where $\epsilon$ represents a normalized intrinsic efficiency which behaves as a constant value inside the fiber. If this efficiency is not exactly 1.0 , the total coupling efficiency would be the product of the intrinsic coupling efficiency and the diffraction efficiency of the holograms. In general, diffraction efficiencies of $\sim 70 \%$ have been reported for transmission holograms, while in reflection the efficiency is usually higher. ${ }^{18}$

\section{Numerical Estimates}

For numerical analysis of Eqs. (27)-(28) we use the following parameters:

Dielectric permittivity of the core of the fiber: $\epsilon_{1}=$ 2.3104
Dielectric permittivity of the cladding of the fiber: $\epsilon_{2}=2.25$.

Total radius of the fiber: $R_{2}=50.0 \mu \mathrm{m}$.

Radius of the core of the fiber: $R_{1}=2.0 \mu \mathrm{m}$.

Coupling constant of the fiber: $\Delta=0.01$.

All these parameters are standard for single-mode fibers.

Wavelength of the incoming radiation: $\lambda=0.8 \mu \mathrm{m}$. With this particular value in the IR region of the electromagnetic spectrum the eikonal condition holds.

We have assumed a plane polarized monochromatic wave for the reference beam defined in the $x-z$ plane as

$$
U_{R 1}=|\mathbf{R}| \exp \left(i \mathbf{K}_{R} \cdot \mathbf{r}\right),
$$

with

$$
\mathbf{K}_{R}=K(\sin \gamma, 0, \cos \gamma) ; \mathbf{r}=(X, 0, Z) .
$$

The angle of incidence $\gamma$ of the reference wave has been fixed at $\gamma=10^{\circ}$ (see Fig. 3).

We have calculated all possible values of $P$ and $S$ for each fixed value of the phase: $\mathbf{K}_{R} \cdot \mathbf{r}$. That is, $P$ and $S$ are functions which depend on the parameters of the eikonal phase:

$$
P, S=f\left(\mathbf{K}_{R} \cdot \mathbf{r}\right) .
$$

We have studied a series of distributions of $P$ and $S$ for different fixed values of the vertical coordinate $X_{2}$ located at the aperture of fiber 2. These values of $X_{2}$ range from $X_{2}=0 \mu \mathrm{m}$, the axial point inside the core, to $X_{2}=50 \mu \mathrm{m}$ for the cladding.

Figure 4 displays the results obtained for $P$ (lower) and $S$ (upper) inside the core for three different $X_{2}$ values: (a) $X_{2}=2 \mu \mathrm{m}$, (b) $X_{2}=1 \mu \mathrm{m}$, (c) $X_{2}=0 \mu \mathrm{m}$. For these three points coupling is assured. Only at the boundary of the core, $2 \mu \mathrm{m}$, is the coupling more critical. The closer to the axis of the fiber the better the coupling.

In Fig. 5 we display the results for the $P$ (bottom) and $S$ (top) distributions at the cladding region with the following specific values: (a) $X_{2}=50 \mu \mathrm{m}$; (b) $X_{2}=$ $40 \mu \mathrm{m}$; and (c) $X_{2}=30 \mu \mathrm{m}$. We observe that for case (a) corresponding to the external boundary of the fiber, $P$ and $S$ coincide, and obviously the coupling is not assured. For the other two cases the coupling condition holds. For four additional values $(3,14,16$, and 32 $\mu \mathrm{m})$ for $X_{2}$, not displayed here, no coupling was obtained. This demonstrates the irregular character of the coupling condition in the cladding.

If we perform an integration of both $P$ and $S$ functions with respect to the vertical variable $X_{2}$ we obtain the coupling condition for the aperture of the entire fiber. This result is equivalent to scanning across the aperture of fiber 2 .

We denote

$$
\begin{aligned}
& \text { INTP }=\int_{-R_{2}}^{+R_{2}} d X_{2} P\left(X_{2}\right), \\
& \text { INTS }=\int_{-R_{2}}^{+R_{2}} d X_{2} S\left(X_{2}\right) .
\end{aligned}
$$

The two integrals have been numerically evaluated. A 


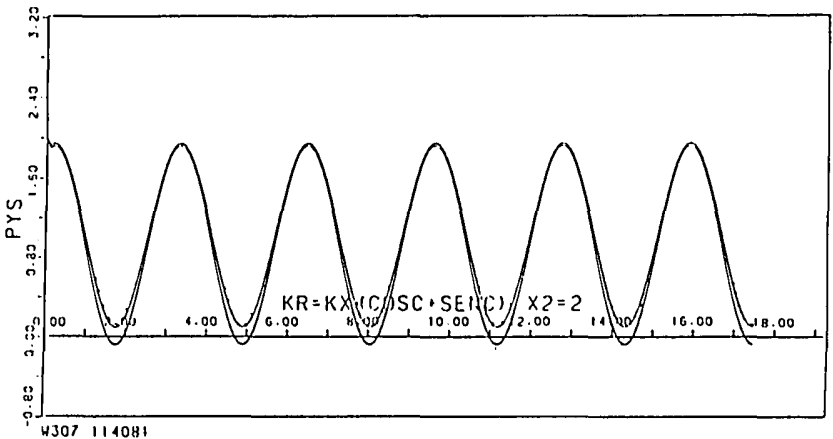

(a)

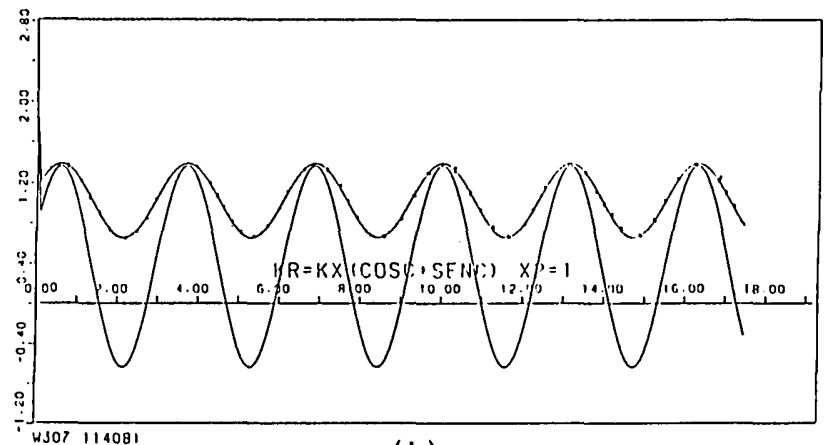

(b)

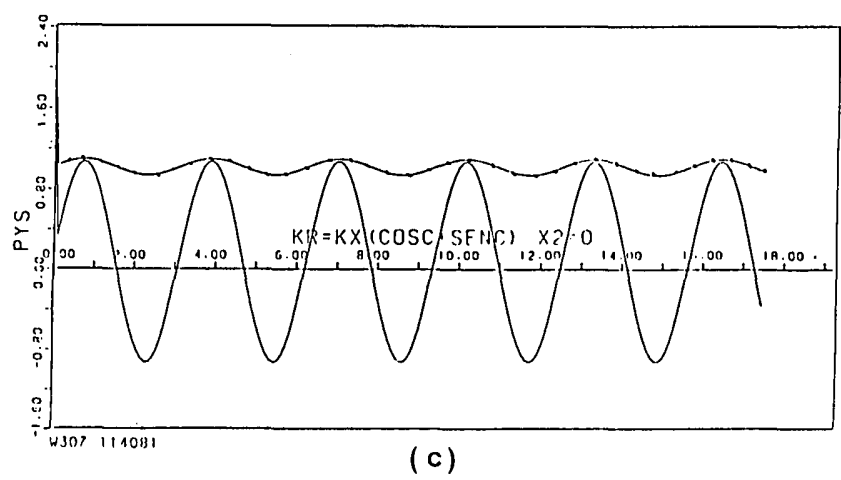

Fig. 4. Coupling condition inside the core of the optical fiber: (a) $X_{2}=2 \mu \mathrm{m}$; (b) $X_{1}=1 \mu \mathrm{m}$; (c) $X_{2}=0 \mu \mathrm{m}$. The upper curve represents the $S$ function, the lower the $P$ function.

mathematical analysis of these two integrals is given in Appendix B. The numerical results are shown in Fig. 6 . We observe that the coupling condition is maintained for the particular state of polarization of the reference beam.

These last results have been obtained similarly for other position vectors:

$$
\begin{aligned}
& \mathbf{K}_{R} \cdot \mathbf{r}=K X \sin \gamma, \\
& \mathbf{K}_{R} \cdot \mathbf{r}=K Z \cos \gamma,
\end{aligned}
$$

and for other values for the angle of inclination $\gamma$ as well. The results are not displayed for simplicity.

\section{v. Conclusions}

Based on the eikonal approximation for the scattering of a TM wave by an optical fiber and a specific

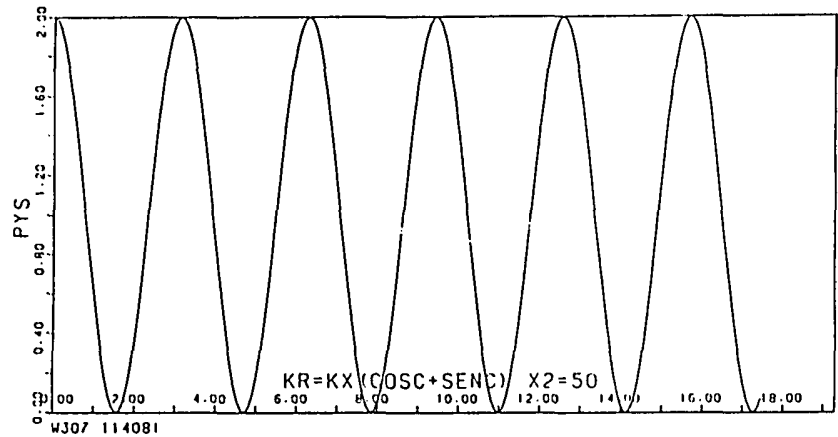

(a)

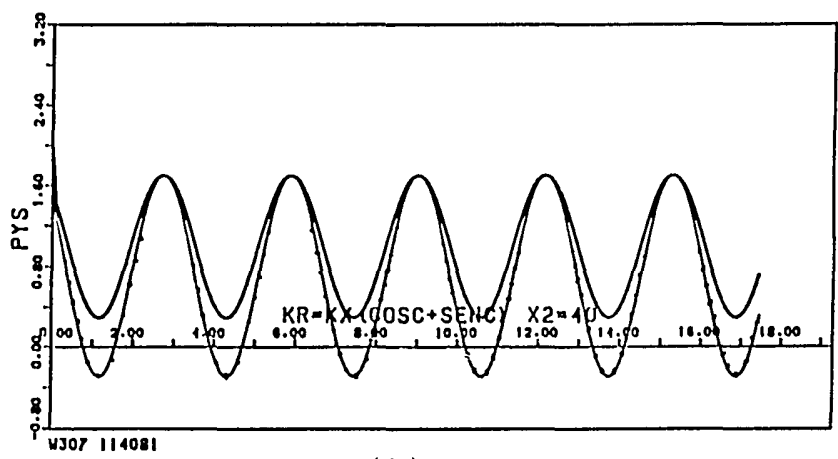

(b)

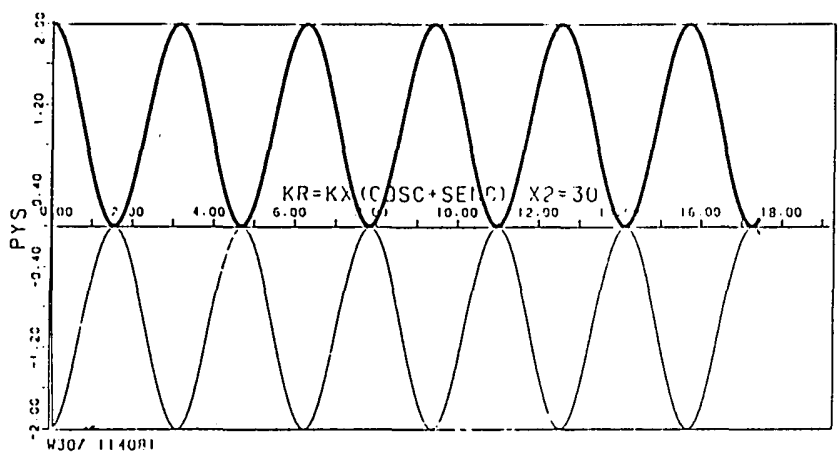

(c)

Fig. 5. Coupling condition inside the cladding and outside the core: (a) $X_{2}=50 \mu \mathrm{m}$; (b) $X_{2}=40 \mu \mathrm{m}$; (c) $X_{2}=30 \mu \mathrm{m}$.

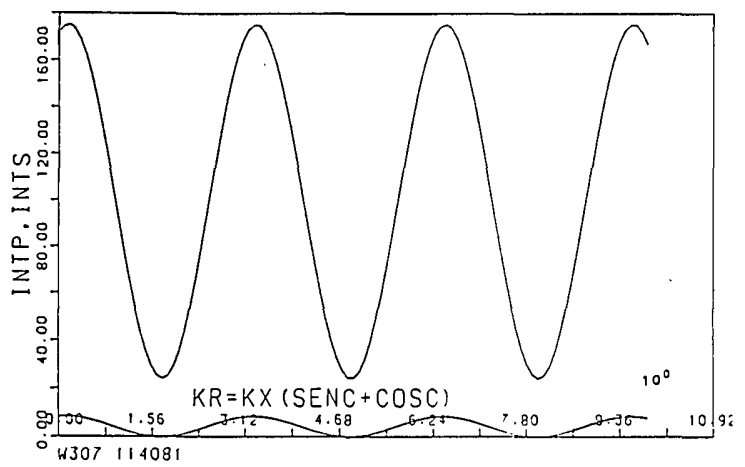

Fig. 6. Integrals of functions $S$ (top) and $P$ (bottom) describing the coupling condition across the $X_{2}$ variable at the entrance face of fiber 2 (as in the scheme displayed in Fig. 3). The condition has been studied for $\gamma=10^{\circ}$. 
holocoupler device, we have developed a mathematical model to describe the coupling condition in a hololensoptical fiber system. A simple description is derived by assuming the condition necessary for a system of implicit functions to have nontrivial solutions different from zero. The influence in the coupling condition of different particular physical parameters has been studied by numerical simulation, particularly by

(1) assuming the reference wave in the $x-z$ plane and fixed parameters: $\epsilon_{2}, \epsilon_{1}, R_{2}, R_{1}$, and $\lambda$; and

(2) choosing an angle of incidence $\gamma$ and a particular state of polarization for the reference beam. Then the coupling condition holds for almost all points of the entrance pupil of the second fiber. We can exclude only some discrete points outside the core in the cladding region. The parameter $Z_{1}$ (the distance of the aperture plane of fiber 1 up to the plane of the first hologram) influences the coupling condition as a periodic function. This method can be extended for other possible reference beams (not necessarily plane waves) and other optical fiber geometries. Further studies of these subjects are now under development.

Part of this work was financially supported by the CAICYT (Spanish Advisory Commission for Scientific and Technological Developments). Partial results were presented at the International Symposium on the Technologies for Optoelectronics, 16-19 Nov. 1987, Cannes, France, at the Conference Laser Technologies in Industry, 6-8 June 1988, Porto, Portugal, and at the HOSPIA Meeting, 12-18 Dec. 1988, Madras, India. We are indebted to O. D. D. Soares from Porto University for helpful suggestions and discussions and to $\mathrm{P}$. K. Mondal for his advice. where $X_{21}=X_{2}^{\prime}$ is the vertical coordinate defined at the aperture pupil of fiber $1, X_{22}=X_{2}$ is the vertical coordinate defined at the aperture pupil of fiber 2 , and

$$
\begin{aligned}
F\left(X_{21}, X_{22}\right)= & 2 R^{2}\left\{1-\cos \left[\varphi_{\text {eik } 1}\left(X_{21}\right)\right]\right\} \\
& \times\left\{\cos \left[-\varphi_{\text {eik } 2}\left(X_{22}\right)+2 \mathbf{K}_{R} \cdot \mathbf{r}\right]\right. \\
& \left.-\cos \left(2 \mathbf{K}_{R} \cdot \mathbf{r}\right)+\cos \left[\varphi_{\text {eik2 }}\left(X_{22}\right)\right]-1\right\} \\
& -\cos \left[\varphi_{\text {eik } 2}\left(X_{22}\right)\right]+1, \\
G\left(X_{21}, X_{22}\right)= & 2 R^{2}\left\{1-\cos \left[\varphi_{\text {eik } 1}\left(X_{21}\right)\right]\right\} \\
& \times\left\{\sin \left[-\varphi_{\text {eik2 }}\left(X_{22}\right)+2 \mathbf{K}_{R} \cdot \mathbf{r}\right]\right. \\
& \left.-\sin \left(2 \mathbf{K}_{R} \cdot \mathbf{r}\right)+\sin \left[\varphi_{\text {eik2 }}\left(X_{22}\right)\right]\right\} \\
& -\sin \left[\varphi_{\text {eik2 }}\left(X_{22}\right)\right] .
\end{aligned}
$$

The necessary and sufficient condition for the system (A3) [together with Eqs. (A4)-(A5)] to have a nontrivial solution different from zero is that the Jacobian be different from zero ${ }^{19}$ :

$$
J=\left|\begin{array}{ll}
\partial F / \partial X_{21} & \partial F / \partial X_{22} \\
\partial G / \partial X_{21} & \partial G / \partial X_{22}
\end{array}\right| \neq 0 .
$$

We obtain immediately the following trivial conditions:

$$
R^{2}, \quad d \varphi_{\text {eik } 1}\left(X_{21}\right) / d X_{21}, \quad d \varphi_{\text {eik } 2}\left(X_{22}\right) / d X_{22}, \quad\left|U_{F 1}\right|^{2} \neq 0
$$

together with

$$
\cos \left[\varphi_{\text {eik1 }}\left(X_{2}\right)\right] \neq-1 \Longrightarrow\left|U_{F 1}\right|^{2} \neq 4
$$

as a coupling condition for the diffracted signal located at the aperture pupil of fiber 1 , and

$$
\begin{gathered}
2 R^{2}\left\{1-\cos \left[\varphi_{\text {eik1 }}\left(X_{21}\right)\right]\right\}\left\{\cos \left[-\varphi_{\text {eik2 }}\left(X_{22}\right)+2 \mathbf{K}_{R} \cdot \mathbf{r}\right] \cos \left(2 \mathbf{K}_{R} \cdot \mathbf{r}\right)+\right. \\
\cos \left[-\varphi_{\mathrm{eik} 2}\left(X_{22}\right)+2 \mathbf{K}_{R} \cdot \mathbf{r}\right]-\cos \left[\varphi_{\mathrm{eik} 2}\left(X_{22}\right)\right] \cos \left(2 \mathbf{K}_{R} \cdot \mathbf{r}\right)-\cos \left[\varphi_{\mathrm{eik} 2}\left(X_{22}\right)\right]+ \\
\sin \left[-\varphi_{\mathrm{eik} 2}\left(X_{22}\right)+2 \mathbf{K}_{R} \cdot \mathbf{r}\right] \sin \left(2 \mathbf{K}_{R} \cdot \mathbf{r}\right)-\sin \left[\varphi_{\mathrm{eik} 2}\left(X_{22}\right)\right] \sin \left(2 \mathbf{K}_{R} \cdot \mathbf{r}\right)- \\
\cos \left[\varphi_{\mathrm{eik} 2}\left(X_{22}\right)\right]\left\{-1+\cos \left[-\varphi_{\mathrm{eik} 2}\left(X_{22}\right)+2 \mathbf{K}_{R} \cdot \mathbf{r}\right]\right\}- \\
\sin \left[\varphi_{\mathrm{eik} 2}\left(X_{22}\right)\right] \sin \left[-\varphi_{\mathrm{eik} 2}\left(X_{22}\right)+2 \mathbf{K}_{R} \cdot \mathbf{r}\right]+\cos \left[-\varphi_{\mathrm{eik} 2}\left(X_{22}\right)+2 \mathbf{K}_{R} \cdot \mathbf{r}\right]-1 \neq 0 .
\end{gathered}
$$

\section{Appendix A: Analysis of the System of Eqs. (23)-(25)}

We shall consider the reference wave as a plane polarized monochromatic wave defined as

$$
U_{R 1}=|\mathbf{R}| \exp \left(i \mathbf{K}_{R} \cdot \mathbf{r}\right)
$$

If we substitute Eqs. (24)-(25) into Eq. (23),

$$
\begin{array}{r}
2 R^{2} \exp \left[2 i \mathbf{K}_{R} \cdot \mathbf{r}\right]\left\{1-\cos \left[\varphi_{\text {eik1 } 1}\left(X_{21}\right)\right]\right\} \times \\
\left\{\exp -\left[i \varphi_{\text {eik2 }}\left(X_{22}\right)\right]-1\right\}= \\
\left.\left\{1-2 R^{2}\left[1-\cos \left[\varphi_{\text {eik1 }}\left(X_{21}\right)\right]\right]\right\} \exp \left[i \varphi_{\text {eik } 2}\left(X_{22}\right)\right]-1\right\},
\end{array}
$$

so that the system of implicit equations to be solved is

$$
\begin{aligned}
& F\left(X_{21}, X_{22}\right)=0, \\
& G\left(X_{21}, X_{22}\right)=0,
\end{aligned}
$$

From Eqs. (A3)-(A5) we obtain

$$
\begin{aligned}
\cos \left[\varphi_{\text {eik } 2}\left(X_{22}\right)\right]= & 2 R^{2}\left\{1-\cos \left[\varphi_{\text {eik } 1}\left(X_{21}\right)\right]\right\} \\
& \times\left\{\cos \left[-\varphi_{\text {eik2 }}\left(X_{22}\right)+2 \mathbf{K}_{R} \cdot \mathbf{r}\right]\right. \\
& \left.-\cos \left(2 \mathbf{K}_{R} \cdot \mathbf{r}\right)+\cos \left[\varphi_{\text {eik2 }}\left(X_{22}\right)\right]-1\right\}+1, \\
\sin \left[\varphi_{\text {eik2 }}\left(X_{22}\right)\right]= & 2 R^{2}\left\{1-\cos \left[\varphi_{\text {eik } 1}\left(X_{21}\right)\right]\right\} \\
& \times\left\{\sin \left[-\varphi_{\text {eik2 }}\left(X_{22}\right)+2 \mathbf{K}_{R} \cdot \mathbf{r}\right]\right. \\
& \left.-\sin \left[2 \mathbf{K}_{R} \cdot \mathbf{r}\right]+\sin \left[\varphi_{\text {eik2 }}\left(X_{22}\right)\right]\right\} .
\end{aligned}
$$

By substituting Eqs. (A9) and (A10) into Eq. (A8) we obtain two solutions:

$$
R^{2},\left|U_{F 1}\right|^{2} \neq 0
$$

which is a trivial condition, and 


$$
\begin{array}{r}
\cos \left[\varphi_{\mathrm{eik} 2}\left(X_{2}\right)\right]+\cos \left[-\varphi_{\mathrm{eik} 2}\left(X_{2}\right)+2 \mathbf{K}_{R} \cdot \mathbf{r}\right] \neq \\
(1 / 2)\left\{\cos 2\left[-\varphi_{\mathrm{eik} 2}\left(X_{2}\right)+\mathbf{K}_{R} \cdot \mathbf{r}\right]+\cos \left(2 \mathbf{K}_{R} \cdot \mathbf{r}\right)\right\}+1,
\end{array}
$$

which is the nontrivial condition for the system to have a solution different from zero and represents the coupling condition formulated in Eq. (26).

\section{Appendix B: Analysis of the Integrals Eqs. (33) and} (34)

The functions $P$ and $S$ are both symmetric with respect to the variable $X_{2}$ :

$$
\begin{aligned}
\operatorname{INT} P & =\int_{-R_{2}}^{+R_{2}} P\left(X_{2}\right) d X_{2} \\
& =2\left[\int_{0}^{+R_{1}} P\left(X_{2}\right) d X_{2}+\int_{+R_{1}}^{+R_{2}} P\left(X_{2}\right) d X_{2}\right],
\end{aligned}
$$

and

$$
\begin{aligned}
\operatorname{INTS}= & \int_{-R_{2}}^{+R_{2}} S\left(X_{2}\right) d X_{2}=2\left[\int_{0}^{+R_{1}} S\left(X_{2}\right) d X_{2}\right. \\
& \left.+\int_{+R_{1}}^{+R_{2}} S\left(X_{2}\right) d X_{2}\right]
\end{aligned}
$$

According to Eqs. (27) and (28) and by substituting the eikonal phase as given in Eqs. (9) and (10) into Eqs. (B1) and (B2) we obtain

$$
\begin{aligned}
\int_{0}^{+R_{1}} P\left(X_{2} d X_{2}=\right. & \int_{0}^{+R_{1}} \cos \left[\varphi_{\text {eik2 }}\left(X_{2}\right)\right] d X_{2} \\
& +\int_{0}^{+R_{1}} \cos \left[-\varphi_{\text {eik } 2}\left(X_{2}\right)+2 \mathbf{K}_{R} \cdot \mathbf{r}\right] d X_{2}, \\
\int_{0}^{+R_{1}} S\left(X_{2}\right) d X_{2}= & \int_{0}^{+R_{1}}(1 / 2) \cos 2\left[-\varphi_{\text {eik2 }}\left(X_{2}\right)+\mathbf{K}_{R} \cdot \mathbf{r}\right] d X_{2} \\
& \times \int_{0}^{+R_{1}}\left\{(1 / 2) \cos \left[2 \mathbf{K}_{R} \cdot \mathbf{r}\right]+1\right\} d X_{2},
\end{aligned}
$$

and similarly for the other interval $\left(+R_{1},+R_{2}\right)$.

\section{References}

1. P. K. Tien, "Integrated Optics and Wave Phenomena," Rev. Mod. Phys. 49, 361-420 (1977); see also J. Gowar, Optical Communication Systems (Prentice-Hall, Englewood Cliffs, NJ, 1984).

2. O. D. D. Soares, "Holographic Coupler for Fiber Optics," Opt. Eng. 20, 740-745 (1981).

3. E. A. Ash, E. Seaford, O. D. D. Soares, and K. S. Pennington, "Holographic Coupler for Integrated Optics," Appl. Phys. Lett. 24, 207-208 (1974).

4. H. Nishihara, S. Inohara, T. Suhara, and J. Koyama, "Holocoupler: a Novel Coupler for Optical Circuits," IEEE J. Quantum Electron. QE-11, 794-796 (1975).

5. G. Goldmann and H. H. Witte, "Holograms as Optical Branching Elements," Opt. Quantum Electron. 9, 75-78 (1977).

6. T. Yoshino, T. Kubota, and T. Ose, "Holographic Couplers for Monomode Fiber," Appl. Opt. 22, 1800-1801 (1983).

7. M. P. Jordan, R. Kompfner, C. J. R. Sheppard, and L. Solymar, "Coupling of Optical Fibers by Means of Volume Holograms," in Proceedings, Sixth European Microoptic Conference, Rome, Italy (1976), pp. 438-442.

8. A. Kogelnik and T. P. Sosnowski, "Holographic Thin Film Couplers," Bell Syst. Tech. J. 49, 1602-1698 (1970).

9. L. Solymar and D. J. Cooke, Volume Holography and Volume Gratings (Academic, New York, 1981).

10. L. De Pedraza and M. L. Calvo, "Non Linear Effects in the Holographic Register: an Improved Alternative to Kozma's Model," Optik 72, 43-49 (1986).

11. A. M. P. P. Leite and O. D. D. Soares, "Holographic Coupler for Fiber Optics Communications," Proc. Soc. Photo-Opt. Instrum. Eng. 213, 10-17 (1980).

12. A. M. P. P. Leite, O. D. D. Soares, and E. A. Ash, "Optical Fiber Bundle Holographic Coupler," Microwaves Opt. Acoust. 2, 4559 (1978).

13. R.F. Alvarez-Estrada, M.L. Calvo, and P. Juncos, "Scattering of TM Waves by Dielectric Fibers: Iterative and Eikonal Solutions," Opt. Acta 27, 1367-1378 (1980).

14. R. J. Glauber, Lectures in Theoretical Physics, Vol. 1, W. E. Brittin and L. G. Dunham, Eds. (Interscience, New York, 1959).

15. L. S. Watkins, "Scattering from Side-Illuminated Clad Glass Fibers for Determination of Fiber Parameter," J. Opt. Soc. Am. 64, 767-772 (1974).

16. M. L. Calvo and P. Juncos, "Bidimensional Scattering of TM Waves by an Optical Fiber: Eikonal Approximation," Proc. Soc. Photo-Opt. Instrum. Eng. 213, 35-37 (1980).

17. O. D. D. Soares, A. M. P. P. Leite, and E. A. Ash, AGARD Conf. Proc. 219, 44.1-44.13 (1977).

18. H. Kogelnik, "Coupled Wave Theory for Thick Hologram Gratings," Bell Syst. Tech. J. 48, 2909-2947 (1969).

19. J. Rey Pastor et al., Análisis Matemático, Vol. 2 (Kapelusz, Buenos Aires, 1958). 